\title{
HIV antibody profiles in serum and CSF of patients with neurological disease can serve as predictors of outcome
}

\author{
Anita Desai, V Ravi, P Satishchandra, N Khanna, Shivaji Rao, M Gourie-Devi, \\ A Chandramuki
}

\begin{abstract}
The serum and CSF antibody profiles were investigated in 100 patients with HIV in relation to the type and severity of neurological disease. Among them, 87 were positive for anti-HIV antibodies in the CSF. In 30 of 87 patients detailed analysis by western blot could be performed. In 20 of 30 the profiles were dissimilar, with more of bands being found in the serum than in the CSF. The correlation of western blot profiles to the clinical outcome indicated that the number of anti-HIV antibody bands as well as the index in the CSF of fatal cases were significantly less compared with non-fatal cases $(p=0.019$ and $p=0.039$ respectively).

(F Neurol Neurosurg Psychiatry 2000;68:86-88)
\end{abstract}

Neurovirology,

Neurology,

Neuromicrobiology,

and Biostatistics,

National Institute of

Mental Health and

Neuro Sciences

(NIMHANS),

Bangalore 560029,

India

A Desai

V Ravi

Department of

Neuromicrobiology

N Khanna

A Chandramuki

Department of

Neurology

P Satishchandra

M Gourie-Devi

Department of

Biostatistics

S Rao

Correspondence to: Dr V Ravi, Department of

Neurovirology, NIMHANS,

Bangalore 560029, India

email vravi

@nimhans.ikar.nic.in

Received 25 August 1998 and in revised form

13 April 1999

Accepted 28 May 1999
Keywords: HIV/AIDS; western blot; neurological manifestations; predictors

Human immunodeficiency virus (HIV) infection was first detected in India during 1985-6. Since then the seropositivity rate has been steadily on the increase and at present it is 22/1000 samples tested. ${ }^{1}$ There are very few studies on the neuropsychiatric aspects of HIV infection in India. In a study conducted 5 years ago at our centre, Desai et $a l^{2}$ reported a seropositivity rate of $1.2 \%(3 / 247)$ among patients with neurological disorders. However, since then there has been a dramatic increase in the number of HIV positive patients presenting with neurological and psychiatric manifestations and in the year 1998 the seropositivity rate was $14 \%$ (80/573). Although some reports pertaining to the various types of neurological manifestations seen in HIV/AIDS are available, there are very few studies which have attempted to search for prognosticators of neurological morbidity in AIDS. The frequent presence of HIV or its genome in the CSF, increased concentrations of IgG, and the presence of oligoclonal bands in the CSF and detection of HIV p24 antigen in the CSF have been described in both symptomatic and asymptomatic subjects infected with HIV..$^{3-7}$ As it would be of considerable clinical value to evolve laboratory predictors for the outcome of patients with and without neurological involvement, so that antiretroviral therapy and other interventions can be initiated early, we evaluated the role of serum and CSF antibody profiles as predictors of outcome in patients infected with HIV. In this paper we present our results in relation to the type and severity of neurological disease and outcome in HIV positive patients.

\section{Materials and methods}

PATIENTS

A total of 3230 patients seen between 1989 and 1997 at the NIMHANS hospital with neurological signs and symptoms underwent HIV testing and $189(5.9 \%)$ were found to be HIV positive. Among them 100 patients with neurological manifestations in whom a CSF sample was available for HIV testing constituted the study group. All these patients had voluntarily consented for HIV testing and were counselled pretest and post-test

\section{HIV ANTIBODY PROFILES}

Coded serum and CSF samples were initially tested for the presence of HIV antibodies using either an enzyme linked immunosorbent assay (ELISA) (Innogenetics Inc, USA, UBI Magiwell, USA and Detect Biochem, Canada) or rapid immunoassay (Immunocomb, Israel, HIV spot, USA) kits supplied under the National AIDS control programme. Reactive samples were retested twice using different kits before considering them as positive. Among those positive, 30 pairs of serum and CSF samples (where sufficient amount of sample was available) were taken up for detailed analysis by western blot using a commercial HIV 1 and 2 kit (GeneLabs, USA). Briefly, $2.5 \mathrm{ml}$ of 1: 50 diluted serum or CSF sample was added onto nitrocellulose strips (containing the electrophoresed and transferred viral proteins) and incubated on a rocking platform for 2 hours at room temperature, washed thrice, and incubated with secondary antibody for 1 hour at room temperature on a rocking platform. Subsequently the strips were washed thrice and bands visualised by adding the substrate. The results were recorded by two independent 

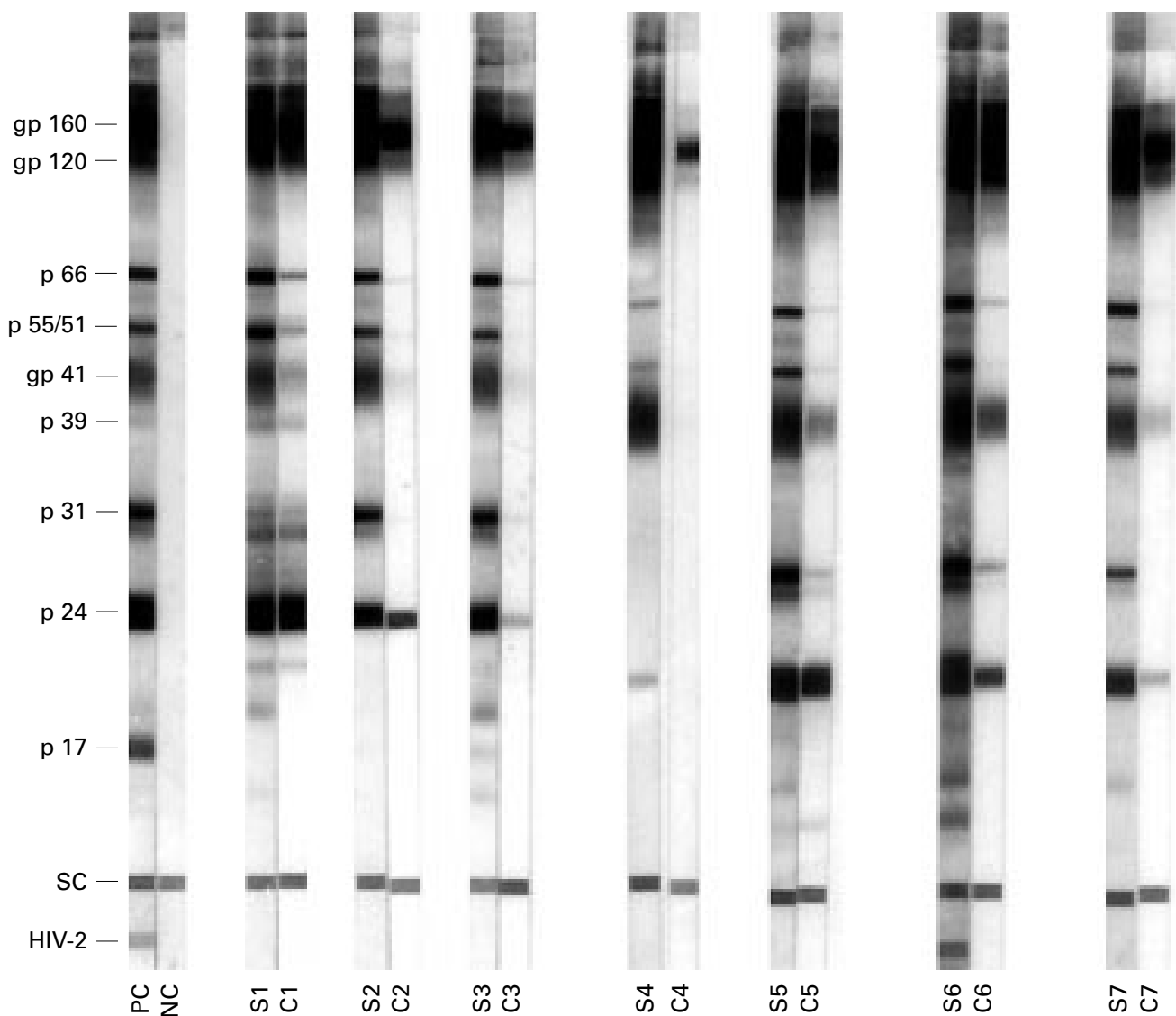

Representative anti-HIV antibody profiles obtained by western blot in serum and CSF. The first two blots on the left were reacted with positive (PC) and negative (NC) control sera provided by the manufacturers of the kit. Lanes S1/C1 to $S 7 / C 7$ represent antibody patterns in serum $(S)$ and CSF $(C)$ obtained from patients infected with HIV The number of bands obtained in the CSF (lanes C1-C7) were less in most patients compared with the corresponding serum samples (S1-S7). Note that the HIV-2 band obtained in the serum (S6) was absent in the corresponding CSF sample of one patient (C6).

investigators ( $\mathrm{AD}$ and $\mathrm{VR})$ blind to the code of the sample. The results were also expressed as an index using a graded scoring system provided by the manufacturer of the kit. In this system the presence of each HIV-1 viral protein band was given the following score: gp 160 or gp $41=1$; gp $120=1$, p 66 , or p 51 , or p $24=0.5$; $\mathrm{p} 31$ or $\mathrm{p} 17=0.25$. Thus the maximum index that could be assigned to any HIV-1 positive sample would be 3.25 .

\section{Results}

CLINICAL FINDINGS

Among the $100 \mathrm{HIV}$ positive patients most of them were men $(93 \%)$ in the age group of $18-60$ years (mean $=32.2$ years) and heterosexual contact was the major risk factor for acquiring HIV infection reported in $80 \%$. Only 30 patients underwent detailed analysis by western blot and in the remaining 70 it could not be carried out because the CSF sample was not adequate. The clinical presentation in all but one of the 30 patients was opportunistic infections of the CNS. These were cryptococcal meningitis $(n=9)$, neurotuberculosis $(n=6)$, toxoplasmosis $(n=3)$, cryptococcal meningitis with neurotuberculosis $(n=3)$, cryptococcal meningitis with toxoplasmosis $(n=2)$, neurotuberculosis with toxoplasmosis $(n=4)$, and herpes encephalitis $(n=2)$. Among these 30 patients 16 succumbed and the remaining 14 recovered from opportunistic infections.

HIV ANTIBODY PROFILES

Among the 100 patients whose serum samples were positive for HIV antibodies by ELISA or rapid immunoasays in 87 patients anti-HIV antibodies were detected in the CSF. In 29 of 30 patients in whom western blot analysis was carried out antibodies to HIV-1 proteins were detected whereas in one reactivity to both HIV 1 and 2 antigens was noted. Antibody profiles obtained in the 20/30 patients were dissimilar in serum and CSF, with more bands being found in the serum than in the CSF (figure). However, in one of these 20 patients a p51 band was seen in the CSF but not in the serum. Antibodies to the HIV-1 glycoproteins (gp160, gp 120, and gp 41) and p66 were seen in almost all the 30 serum and CSF samples. However, antibodies to three other HIV-1 proteins- p55, p39, and p17-were seen significantly less often in the CSF than in the serum. In the lone patient who was positive for both HIV-1 and HIV-2 antibodies the band to HIV gp 36 was detectable in the serum but not in the CSF.

Correlation of the mean levels of HIV antibody bands obtained in the western blot with the clinical outcome is presented in the table. The number of HIV-1 bands as well as 
Correlation of HIV antibody profiles with outcome in patients with neurological manifestations

\begin{tabular}{lccl}
\hline Variable & $\begin{array}{l}\text { Fatal } n=16 \\
\text { Mean }(S D)\end{array}$ & $\begin{array}{l}\text { Non-fatal } n=14 \\
\text { Mean }(S D)\end{array}$ & p Value* \\
\hline Serum bands & $63.97(13.05)$ & $68.90(14.98)$ & $0.34(\mathrm{NS})$ \\
CSF bands & $43.16(20.64)$ & $60.12(17.29)$ & $0.02(\mathrm{SIG})$ \\
Serum index $\dagger$ & $3.09(0.25)$ & $3.12(0.13)$ & $0.68(\mathrm{NS})$ \\
CSF index† & $2.40(1.06)$ & $3.01(0.25)$ & $0.04(\mathrm{SIG})$ \\
\hline
\end{tabular}

*A Student's $t$ test was used.

†The index represents the mean of the indices obtained with individual samples based on the criteria prescribed by the manufacturer of the kit (details provided in Materials and Methods section).

the index value in the CSF of fatal cases were significantly less compared with the non-fatal cases. However this was not true for antibody profiles obtained in the serum between the two groups.

\section{Discussion}

Patients with HIV infection may manifest neurological symptoms during the initial stages of infection. Abnormalities in the CSF such as increased white blood cell count, increased total protein concentrations, presence of HIV p24 antigen, and isolation of HIV have been reported in patients with and without neuropsychiatric findings. ${ }^{78}$ Some earlier studies have suggested that HIV antibody patterns can been used to prognosticate the evolution of HIV related CNS disease, although not for predicting the outcome of patients with neurological disease. Whereas it has been suggested by some that studying the evolution of the immune response to HIV antigens in the CSF may serve as a useful marker for CNS disease progression $^{39}$ others have refuted such a claim. ${ }^{10}$ In this study we have shown that antibody profiles in the serum and CSF of HIV positive patients with neurological symptoms are quite different in most $(70 \%)$ of the patients. The probable reasons for this are twofold; most of the patients $(27 / 30)$ were in the advanced stage of HIV infection when opportunistic infections of the CNS predominate. It is possible that in view of the overwhelming nature of the opportunistic infection of the CNS the HIV specific immune responses could have been blunted within the neural compartment. Alternatively, it is possible that the antigenic stimulus after viral expression within the CNS is distinct compared with the systemic compartment resulting in different HIV antibody profiles. In the event of a breach in the blood-brain barrier, spillover of serum into the CSF compartment is likely to result in identical HIV antibody profiles between the two compartments. However in 11 of 18 of our patients in whom such a breach was suspected (using a crude index of CSF: serum albumin ratios) we found differences in HIV antibody profiles in serum and CSF, This probably suggests that there is differential processing of HIV antigens within the two compartments.

The number of bands and the reactive indices in the CSF were significantly different between patients who died and those who did not $(p=0.019$ and 0.039 respectively) in the CSF. It is likely that the poorer prognosis of the pateints with a blunted antibody profile in the CSF is a mere reflection of the advanced immunodeficiency. Such a difference was, however, not noted between those who died and those who did not with respect to serum samples $(p=0.343$ and 0.684 respectively). These results suggest that HIV antibody profiles could serve as predictors of final clinical outcome in patients with HIV with neurological manifestations (table). However a larger, prospective, longitudinal study of HIV antibody profiles in serum and CSF must be carried out in various categories (with and without opportunistic infections of the nervous system) to draw firm conclusions. Such longitudinal studies would enable identification of the changing patterns of immune response within the CNS. A study of such patterns could in turn predict the evolution of distinct "neurotropic" immune escape variants if any within the CNS.

We acknowledge the support provided by the National AIDS Control Organisation, Ministry of Health and Family Welfare, Government of India. The technical help provided by $\mathrm{Mr}$ Mahesh is also gratefully acknowledged.

1 National AIDS Control Organisation. HIV/AIDS in India. In: Nexus, Newsletter focusing on Child and Reproductive Health issues, STDs and AIDS. New Delhi: Population Services International, 1998:10.

2 Desai A, Puttaram S, Chandramuki A, Ravi V. Seroprevalence of HIV infection at a neuropsychiatric center in South India. F Acquir Immune Defic Syndr Hum Retrovirol 1993;6:534-6.

3 Resnick L, di-Marzo -Veronese F, Schupbach J, et al. Intrablood brain barrier synthesis of HTLV-III specific IgG in patients with neurologic symptoms associated with AIDS or AIDS related complex. N Engl f Med 1985;313:1498or AII

4 Ackerman R, Nekic M, Jergens R. Locally synthesised antibodies in cerebrospinal fluid of patients with AIDS. $f \mathrm{Neu}$ rol 1986;233:140-1.

5 Goudsmit J, Wolters EC, Bakker M, et al. Intrathecal synthesis of antibodies to HTLV-III in patients without AIDS or AIDS related complex. BMF 1986;292:1231-4.

6 Chiodi F, Asjo B, Fenyo E-M, et al. Isolation of human immunodeficiency virus from cerebrospinal fluid of antibody positives carrier without neurological symptoms. Lancet 1986;ii: $1276-7$.

7 McArthur JC, Cohen BA, Farzdegan H, et al. Cerebrospinal fluid abnormalities in homosexual men with and without neuropsychiatric findings. Ann Neurol 1988;23(suppl): S347.

8 Barohn RJ, Gronseth GS, Amato AA, et al. Cerebrospinal fluid and nerve conduction abnormalities in HIV positive fluid and nerve conduction abnormalities

9 Sonnenberg AB, Madeleine AE, Von Syndow MAE, et al. Associating between intrathecal anti-HIV-1 immunoglobuin G synthesis and occurrence of HIV-1 in cerebrospinal fluid. AIDS 1989;3:701-5

10 Goswami KK, Kaye S, Miller R, et al. Intrathecal IgG synthesis and specificity of oligoclonal IgG in patients infected with HIV-1 do not correlate with CNS disease. $\mathcal{F}$ Med Virol 1991;33:106-13. 